tiöned in these columns on September 8. This position lies in Sagittarius about $x_{\frac{1}{2}}^{\circ}$ south-west of the fifthmagnitude star $\omega$, and is on the meridian about 7.30 p.m.

As the magnitude of the comet is given as 13.0 , it is unlikely that observations in these latitudes will be possible for some time, but the southern declination is decreasing, and perihelion passage is not due until January 8, I9II. According to the ephemeris, the comet was at its nearest point to the earth early in August, and its present distance is about 155 million miles.

The Luminosity of Comets.-As a reprint from Science, N.S., vol, xxxii., No. 817 , we have received a paper in which Mr. W. L. Dudley discusses the causes which produce luminosity in cometary bodies. According to the author's reasoning, luminous comets are simply masses of gaseous matter illuminated by the heavy electric discharges constantly flowing from the sun to each of the planets. The "ionic breeze" thus produced renders the comet luminous, and at the same time brushes matter along with it to form the tail. Should the comet get into such a position as to be under the influence of two planets (kathodes) at the same time, more than one tail is produced by the streams of ions from the sun (anode). The author also offers explanations on this hypothesis for the production of multiple heads, the change of direction which sometimes takes place in the tail, and the polarisation of cometary light.

Coloured Stars between the Pole and $60^{\circ} \mathrm{N}$. Declination.-In continuation of previous lists, Herr Krüger now publishes, in No. 444. $\mathrm{I}$ of the Astronomische Nachrichten, a list of coloured stars which lie between the North Pole and $60^{\circ} \mathrm{N}$. declination. The list includes I9 I stars, and for each object gives the catalogue numbers, the colour on the Potsdam scale, Herr Krüger's observed colour, on a scale where $0^{*}=$ white, $\mathbf{1 0}^{2}=$ red, the magnitude and the spectral type. Ninety-three of the stars lie within the colour-limits $W_{G}-G-$, and are of the second and third types.

Observations of the Companion of Sirius.-The observations of the companion of Sirius, made with the 40 -inch refractor by Prof. Barnard during November 30, 1909, to March 15, are recorded in No. 617 of the Astronomical fournal. The values for position-angle and distance, for the mean epoch (19ro.106), are $89.09^{\circ}$ and $9 \cdot 07^{\prime \prime}$ respectively.

The Perseid Shower, 1910.-In a note appearing in No. 617 of the Astronomical Journal Mr. E. F. Sawyer describes the meteor observations made by him at North Weymouth, Mass., on August 9, II, I2, and 13. The hourly rates for one observer, covering one-sixth of the visible sky with the centre in Perseus, were $15,15,8$, and 6 on these respective dates. The meteors generally were bright, and left streaks for one or two seconds; the radiant was found to be at $38.5^{\circ},+55.5^{\circ}$.

A Modified Method for Nadir Observations.-In making the ordinary nadir observations, where a bright thread is made to coincide with its reflected image, there is always some little doubt as to when exact coincidence occurs. To obviate this difficulty, Mr. R. M. Stewart suggests a method where the reflected bright image is obliterated by a dark thread. Experiments made with the Ottawa meridian circle show that this method is simple, and gives greater accuracy than the older one. In making the observation, the bright reflected image and the illuminated thread are brought nearly to coincidence as usual, but the eye-piece, or attached plane mirror, is then slightly rotated so that the field becomes dark and the thread invisible, although the reflected image remain bright, A slight motion then causes the dark thread to occult the bright image, and it is stated that this operation can be performed with much greater certainty than can the bringing into exact coincidence two bright lines (Journal R.A.S. Canada, vol. iv., No. 4).

A New Micrometer.-Dr. Doberck describes a new micrometer in No. 4432 of the Astronomische Nachrichten NO. 2 I36, VOL. 84] made to his specifications by Messrs. Cooke and Son. In this instrument the frame carrying the wires is the only readily movable part, so that there is practically no fear of dragging, and the whole is built very rigidly and strongly, thus obviating any likelihood of derangement.

The errors of the screw, as shown by an investigation involving 4000 settings, are extremely small, the corrections being considerably below the probable errors of setting on stars. The instrument is called the Elizabeth Thompson micrometer.

The Mfan Parallax of Tenth-magnitude Stars.-From a number of measures, made during $1907-9$, of the Engelhardt companions of Bradley stars, Dr. H. E. Lau finds that the mean parallax of tenth-magnitude stars, taking Campbell's value for the sun's velocity, is o.0o10"; this is smaller than the value found by Comstock. The value derived by Kapteyn's method should be $0.00 \mathrm{II}^{\prime \prime}$, so that it would appear that these faint stars are not abnormal in constitution, nor is there indicated any marked absorption in space (Astronomische Nachrichten, No. 4430).

\section{HALLEY METEORS}

[ $\mathrm{N}$ a communication to Dr. W. J. S. Lockyer, Prof. David Todd, of the Amherst College Observatory, writes as follows :-

"So well established is the Schiaparelli-Newton theory of the connection between comets and meteors, that it is highly important to obtain observations from every possible radiant, in order to ascertain whether there may be any that do not conform to the general law.

"Particularly is it desirable to observe the meteors of the Halley stream. Prof. W. H. Pickering directed attention to the possibility of observing such meteors early last May, when the earth was at its nearest, not to the comet, but to the comet's orbit. Many astronomers kept an outlook for these meteors; and on the night of May $5^{-6} I$ made a balloon ascension for this purpose, with a certainty of clear skies, as the spring season had been especially cloudy. However, hardly more meteors than usual on a May night were seen, and none of them appeared traceable to the Halley radiant. Indeed, I had very little expectation of seeing Halley meteors on that occasion, as the region of the cometary orbit then nearest to us was that through which the comet had passed seventy-five years ago.

"Quite different will be the conditions next month. On October 18 the earth is closest to that region of Halley's orbit where the comet passed on March $13-15,1910$, and from which, therefore, it may be expected that many meteors will enter the earth's atmosphere, as the comet has visited that region so recently. Very unfortunately, the moon fulls at just that time, so that all the faintest shooting stars would be lost.

"Probably it will be well to begin the watch as early as October 15, as evidently the meteoric matter in this case is widely scattered along the comet's path.'

According to the Daily Mail of October 4, a very brilliant meteor is recorded to have been observed at Johannesburg on October 3. Perhaps this may be a forerunner of the swarm referred to by Prof. Todd. The account of this large meteor is as follows:-

"Johannesburg, Monday.

"Johannesburg was startled at $8.5^{\circ}$ this evening by the largest shooting star or meteor ever seen in the district.

"Its light was equal to that of a naval searchlight at fifty yards' range. The sky was illumined for three minutes, and the streets were as light as if it were day. Natives were terrified, believing that the end of the world was at hand.

"The astronomical observers at the local observatory were nearly blinded. The director of the observatory estimated that the meteor was only iso miles from the earth. The head of the meteor, he says, was pearshaped and half the size of the moon in diameter. The tail was straight at first, but afterwards turned towards the south.", 